\title{
Consent for Delivery Room Studies: What Can Be Learned from Perceptions of Parents
}

\author{
Maria C. den Boer ${ }^{a, b}$ Mirjam Houtlosser ${ }^{b}$ Ruben S. G. M. Witlox ${ }^{a}$ \\ Henriëtte A. van Zanten ${ }^{a}$ Martine C. de Vries ${ }^{\text {b, }}$ Arjan B. te Pas ${ }^{a}$ \\ aDivision of Neonatology, Leiden University Medical Center, Leiden, The Netherlands; ${ }^{\mathrm{b}}$ Department of Medical \\ Ethics and Health Law, Leiden University Medical Center, Leiden, The Netherlands; 'Department of Pediatrics, \\ Leiden University Medical Center, Leiden, The Netherlands
}

\section{Keywords}

Neonatal resuscitation · Research ethics · Qualitative research

\begin{abstract}
Background: Obtaining ethically valid consent to participate in delivery room (DR) studies from parents facing an imminent premature birth can be challenging. This study aims to provide insight into parental experiences with and perceptions of consent for DR studies. Methods: Semistructured interviews were conducted with parents of very and extreme preterm infants. Interviews were audio-recorded, transcribed, and analyzed using the qualitative data analysis software Atlas. ti V.8.4. Results: Twenty-five parents were interviewed. Despite being in an emotional and stressful situation, most parents considered being approached for DR studies as valuable. According to parents, this was mostly due to appropriate timing and communication, compassion, and investigators not being obtrusive. Interviewed parents generally decided to accept or decline study participation based on perceived risk. Parents differed widely in how risk of specific study interventions was perceived, but agreed on the fact that parental consent is needed for DR studies that
\end{abstract}

Karger@karger.com www.karger.com/neo

Karger"

GOPEN ACCESS
(C) 2022 The Author(s)

Published by S. Karger AG, Basel

This is an Open Access article licensed under the Creative Commons Attribution-NonCommercial-4.0 International License (CC BY-NC) (http://www.karger.com/Services/OpenAccessLicense), applicable to the online version of the article only. Usage and distribution for commercial purposes requires written permission. involve risk. There was no consensus among parents on deferred consent for DR studies running at our NICU. However, parents considered deferred consent appropriate for observational studies. Furthermore, it became clear that parental misunderstanding of various aspects of DR studies, including aims, the concept of randomization, and risk associated with specific interventions, was common. Conclusions: Insight into parental perceptions of consent for DR studies allowed us to determine areas where the validity of parental consent can be improved. Further research on parental perspectives for consent for DR studies will allow us to establish consent procedures that are considered both valid and valuable.

(c) 2022 The Author(s).

Published by S. Karger AG, Basel

\section{Introduction}

Informed consent for research involving human participants is considered a cornerstone in research ethics. The principle of informed consent protects the autonomy of human subjects and is embedded in various ethical codes and regulations, such as the Declaration of Helsinki [1] and the Good Clinical Practice guidelines [2]. In 
order to be valid, consent should be provided voluntarily by a participant, or in well-defined situations by a proxy that is deemed to be mentally competent, has received appropriate information, and is able to understand this information [1-3].

As robust evidence on neonatal resuscitation interventions is lacking [4], conducting delivery room (DR) studies is much needed. Neonates clearly cannot provide a valid consent for these studies, and thus consent for DR studies should be obtained from a proxy with parental responsibility. However, obtaining ethically valid proxy consent for DR studies can be particularly challenging. Investigators need to approach parents facing an imminent premature birth to inform them about possible research participation of their very fragile infant who is not even born yet. If consent is obtained, the validity of consent can be called into question as it is often provided by emotionally distressed parents within a tight timescale for decision-making [5-7]. However, not approaching parents facing an imminent premature birth for the study participation of their infants result in selection bias [8], which is therefore also undesirable.

As part of a research project studying ethical aspects of recording and reviewing neonatal resuscitation [9-12], we conducted interviews with parents of very and extremely premature infants. One of the objectives for the study was to provide insight into parental perceptions of consent for using recordings of neonatal resuscitation for various purposes, including DR studies. By doing so, we gained insight in parental perceptions regarding consent for DR studies. These insights are reported in this study.

\section{Methods}

At the Neonatal Intensive Care Unit (NICU) of the Leiden University Medical Center (LUMC), a tertiary perinatal center with averagely 800 admissions a year, recording neonatal resuscitation is considered standard care and does not require parental consent. Recordings of neonatal resuscitation are used for plenary audits, and parents are offered to review the recordings of their infant. Furthermore, recordings are used for the majority of DR studies conducted at our NICU.

\section{DR Studies at the LUMC}

In accordance with a ruling of the Ethics Review Committee (ERC) of the LUMC, parents are counseled for a maximum of 2 DR studies simultaneously. Parents are generally counseled by a research team consisting of 5-9 investigators (mostly female and $\mathrm{PhD}$ students). DR studies that were running during the study period included a minimal risk study assessing the efficiency of a respiratory functioning monitor (RFM) (MONitoR trial; Dutch Trial Register NTR4104, clinicaltrials.gov NCT03256578), a minimal

Consent for Delivery Room Studies: What

Can Be Learned from Parents risk study assessing the effect of initial high versus low oxygen on breathing effort (IMPROvE study; trial registration number: NTR6878), and 2 subsequent studies assessing the feasibility and effectiveness of physiology-based cord clamping (ABC project; trial registration numbers: NTR7194 and NCT03808051). These DR studies all used recordings of neonatal resuscitation. In addition to these DR studies, various other neonatal trials were running at the NICU.

For the minimal risk MONitoR trial and IMPROvE study, both a prospective and a deferred consent approach could be used. A deferred approach allows investigators to enter neonates in DR study protocols without parental consent. As soon as reasonably possible, parents are informed about their infant's study participation and asked for permission to continue their infant's study participation and to use already obtained data.

\section{Data Collection}

For our study, convenience sampling was applied. All parents that were invited to watch the recordings of their very or extremely preterm infant in the period from February 2018 to October 2019 were approached to participate in our study on ethical dilemmas of recording and reviewing neonatal resuscitation. Parental perspectives on reviewing recordings of neonatal resuscitation, as well as further information about recruitment, are described elsewhere [12]. Interviews were conducted between February 2018 and October 2019. Interviews were conducted by M.C.B. Using semistructured interviews, parents were questioned about their perceptions of consent for the usage of recordings of neonatal resuscitation for DR studies. This resulted in insight in parental perspectives regarding consent for DR studies. Using an iterative approach, new participants were questioned more in depth about their perceptions of consent for DR studies. Parents were asked to reflect on their consent procedure (either antenatal or deferred consent), alternative consent procedures, the most appropriate consent procedure, and the most appropriate timing of consent, amongst others. Inclusion of participants continued until thematic saturation was reached on parental perspectives on recording and reviewing neonatal resuscitation.

Data Analysis

Interviews were audio-recorded and manually transcribed. M.C.B. and M.H. independently coded various transcripts. Data were first analyzed in a process of open coding. During consensus meetings (M.C.B., M.H., and M.C.V.), main themes connected to consent for DR studies emerged. The qualitative data analysis software program Atlas ti (V.8.4) was used for analysis.

\section{Ethics}

This study was reviewed by the ERC of the LUMC. In concordance with laws and guidelines, a statement of no objection against execution of the study was issued by the ERC (P16.316).

\section{Results}

In 13 interviews, 25 parents were interviewed. Parental dyads (all father/mother) were interviewed as a couple. Although not all parents had a Dutch background, all par- 
Table 1. Characteristics interviews ( $n=25$ parents or 13 parental dyads)

\begin{tabular}{|c|c|}
\hline Fathers, $n(\%)$ & $12(48)$ \\
\hline Bereaved, parental dyads, $n(\%)$ & $1(8)$ \\
\hline \multicolumn{2}{|l|}{ Age, median (range) } \\
\hline Mothers & $32(23-41)$ \\
\hline Fathers & $34(24-45)$ \\
\hline \multicolumn{2}{|l|}{ Parity, mothers, $n(\%)$} \\
\hline Nulliparous & $9(69)$ \\
\hline \multicolumn{2}{|l|}{ Mode of delivery, mothers, $n(\%)$} \\
\hline Caesarean section & $8(62)$ \\
\hline Vaginal & $5(38)$ \\
\hline \multicolumn{2}{|l|}{ Resuscitation, infants, $n$ (\%) } \\
\hline CPAP & $19(100)$ \\
\hline PPV & $15(79)$ \\
\hline Intubation & $1(5)$ \\
\hline Cardiac resuscitation & $1(5)$ \\
\hline Gestational age, median (range) & $28+2(24+6$ to $30+3)$ \\
\hline Days between birth and interview, median (range) & $29(10-267)$ \\
\hline \multicolumn{2}{|c|}{ Information provision about DR studies, parental dyads, $n$ (\%) } \\
\hline Not informed about DR studies & $1(8)$ \\
\hline Informed about 1 study & $6(46)$ \\
\hline Informed about 2 DR studies & $5(38)$ \\
\hline Informed using deferred consent & $1(8)$ \\
\hline \multicolumn{2}{|l|}{ Consented, parental dyads, $n(\%)$} \\
\hline Consented to (all) DR studies & $8(62)$ \\
\hline Consented to one, refused another & $3(23)$ \\
\hline Refused all DR studies & $0(0)$ \\
\hline Counseled but no time to consent & $1(8)$ \\
\hline
\end{tabular}

ents were proficient in the Dutch language. All parents had been approached for neonatal research. One parental dyad was not approached for consent for DR studies, and another parental dyad was approached for deferred consent for DR studies. None of the neonates died in the delivery room; however 2 children of 1 parental dyad died during admission to our NICU. Further participant characteristics are listed in Table 1. Five main themes were identified: participating in neonatal research, being approached for consent for DR studies, deferred consent, parental understanding, and risk. Illustrative quotes are displayed in Table 2.

\section{Participating in Neonatal Research}

Interviewed parents reported various perceptions of participating in neonatal research. Parents often acknowledged that their infant(s) could only have received good care during neonatal resuscitation due to previous DR studies that have been conducted and reported altruistic motivations to consent to DR studies. Many interviewed parents related conducting research to improving care and reported considering research as inherent when admitted to a university hospital. Several parents furthermore reported to put their trust in scientific integrity. Al- though all interviewed parents were positive about participating in neonatal research, they also acknowledged that some parents would not be positive about it.

\section{Being Approached for Consent for DR Studies}

For most interviewed parents, being approached antenatally for DR studies was a positive experience. Parents considered information provision about DR studies as a positive distraction in a stressful period, as an opportunity to receive extra information about prematurity, or even as an opportunity to prevent boredom when admitted for a longer period. Furthermore, interviewed parents reported that being approached for more than $1 \mathrm{DR}$ study was not problematic, but several interviewed parents emphasized that one could also demand too much from parents. A few interviewed parents reported that being approached for DR studies was too much because there was already so much going on. Having to think about study participation in such circumstances was considered stressful.

Parents reported various factors that contributed to positive experiences with being counseled for DR studies. Important factors were appropriate communication and compassion. Interviewed parents furthermore highly val- 
Table 2. Illustrative quotes

Perceptions on participating in neonatal research

Father: Of course, so many studies have been conducted in the past, and that's why they could have cared for her so well (...) Mother: Yes, it's like contributing to the next generation, to the children that will be born in the future. LP14

Well, we've been confronted quite a lot with research and things like that. But, this is a university hospital, and eventually all you want to do is to improve and provide the best possible care. LP04 - father

For me, it was quite easy to sign the papers. Although I did not read it all, I felt like, I can trust it is ok. Because I know, it is research, and research needs to meet certain requirements, and well, these requirements were all covered, so yes, ok, I can sign with confidence. LP07 - mother

I could imagine that certain people would not participate in research due to ethical beliefs. LP02 - mother

Perceptions on being approached for consent for delivery room studies

Yes, it is a perfect way to distract the mother. Really! They gave us these leaflets, we read them all. It is the perfect way to distract parents from what really happens. Because as a new parent, as a future parent, you really need to gain an understanding of prematurity. You don't know what to expect. So these studies, they add extra information. LP12 - mother

So there were a few days that I was like, well, starting to get bored. What am I doing here? Well, you know, that's the moment you can explain the study. LP01 - mother

Of course, it was a torrent of information, but it actually helped me to keep on going. Because I have been admitted for three weeks. And I really enjoyed it, it was, somehow you needed to think in a different way. To deal with something else. Something different than my own story. No, it actually really helped me to think about different things and to think about my stay here from another perspective. LP19 - mother

If they would have asked for ten more, we would probably feel like: well, this is kind of enough now! LP01 - mother

Maybe [it could be addressed] at the intake with the midwife. That she's the one who addresses [research]. Like, in case you will deliver prematurely, what do you want in terms of research? Would you like to participate or not? LP27 - father

And maybe the investigator could contact either the physician or the nurse, like, hey, would this be the right moment? LP07 mother

Well, I think the doctor, the pediatrician is the right person to announce it. At least, to introduce it, to tell that there are [studies]. And that there is the possibility that somebody approaches you, or that you can indicate yourself that you are ready to be informed about it. LP14 - father

Of course, you are thinking about what decision to make. Subconsciously it is on your mind. But you already have so much on your mind in such situation. You're constantly thinking about what to do to do well. With everything! Then [research] is just another thing that adds on to the stress. LP16 - father

Aspects that contributed to a positive experience with being counseled

In such situations, I think the compassionate skills from the one asking you the question, yeah, I think she had that skills. She really sat down, what is going on, how are these people doing? Is this the right moment to pose this question? LP14 - father

Well, the way you are approached. Even if the timing is inappropriate, the way you are approached really makes a difference. LP22 mother

Mother: And how it was asked, it was asked in a peacefully manner, very compassionate. We were allowed to think about it. No pressure at all

Father: Especially with this oxygen trial, I found it very pleasant, I think it was the physician who said, if you don't know what to do, just say no, it doesn't matter. LP22

And they explained that it really depends on the situation, and if the situation is different than expected, or if anything goes wrong, they will deviate from the protocol. So, we knew that on beforehand. (...) That research is not conducted at the expense of everything. They explained that very clearly. LP16 - father It was really good that your colleague came and explained everything, instead of solely receiving those leaflets. LP07 - mother

Perceptions on deferred consent

Imagine something would have gone differently than planned. And then afterwards you hear the monitor was blinded, and even though the monitor did not influence anything, I think that's tricky. I would not have been happy in that case. LP07 - mother So, maybe you should obtain informed consent that it is ok not to obtain an informed consent. Do you know what I mean? Explain that this is a university hospital. Would it be ok if we include her in some studies, in case everything goes very fast, and we estimate it will not have any consequences for her? As a parent, it gives you at least some sense of control of what happens. LP11 - mother It really depends on how invasive the study is. You know, just adding an extra pulse oximeter because that one might be more accurate than the one you normally use, well, you know, you don't have to tell me. It's fine to me to just use that data. LP22 - father

Parental misunderstanding

And the other one, what was the other [study]? The placenta, there was also something with the placenta, there was something with the placenta, wasn't it? LP03 - mother 
Table 2 (continued)

Perceptions on risk of delivery room studies

Father: In my opinion, benefits do not outweigh possible risks. Maybe that's sounds a bit strange, but, yeah.

Interviewer: Preferably a study

Father: ... without any risks, but also without any benefits, than a study with possible risks and some benefits. LP07

They probably have thought about it carefully, but for parents it may sound, maybe that's the whole point, it might be scientifically

sound, but $100 \%$ oxygen, that sounds quite scary for us parents. LP1 1 - father

If there is any risk for the child, parents need to consent. LP12 - mother

Perceptions on perceived risk of delivery room studies

Mother: It was more difficult to make a decision, to say yes on her behalf (...)

Father: Because you want to protect her. From things that can go wrong (...) She cannot make the decision. And every decision that I make for her, I really have to support these decisions. I need to have the feeling that this is the right thing to do

LP04

What we considered as a very important aspect is for how long the study has been running already. Because somehow that matters.

Whether the study is running for just one month, or already for a year. Because we know, if something is wrong with the study, the

study will be stopped. So if, the study is already running for a while, you know it already passed that stage. LP16 - mother

But instinctively, for instance with the monitor trial, you switch off the monitor, you take something away. LP07 - mother

And maybe you will lose your child, because you made the decision to participate in that study. I would never forgive myself. I would think by myself, why did I participate at all? LP03 - father

So, and if you see that she is on a ventilator, well, you could say, you made the decision for $100 \%$ [oxygen]. Maybe she has problems with her lungs because they gave her too much at once. LP09 - father

The monitor trial, well, that is easier to reverse. If they say, please turn on the monitor again, or whatever. I guess. I don't know how that happens, monitor on or off. LP11 - mother

ued the fact that investigators were not obtrusive and that they told parents that declining participation was also fine and that research was always of secondary importance. Moreover, parents appreciated to receive additional verbal information to written information. Furthermore, timing of obtaining consent was considered important. Various parents reported the importance of checking with the obstetric staff whether it is an appropriate moment to approach parents. Another possibility to improve the timing of obtaining consent for DR studies reported by several parents was to expedite information provision to earlier in the pregnancy.

\section{Deferred Consent}

Although many parents, including parents approached for deferred consent, agreed that usage of a deferred consent approach could be appropriate, various parents also feared it could undermine confidence in providers or that using this approach could be tricky in case something would have gone wrong during neonatal resuscitation. According to parents, these risks could be reduced by adding a general consent for conducting studies using a deferred consent approach. Among parents, consensus about the appropriateness of deferred consent for DR studies was lacking. However, several parents reported that a deferred or waived consent approach would be appropriate for observational research.

\section{Parental Understanding}

When parents recalled information provided to them during the consent process, parental misunderstanding on aspects of DR studies was frequently identified. For example, several parents misunderstood the concept of randomization, the aim of a study, or specific study interventions. Interviewed parents often were not aware of these misperceptions; however, various parents reported that they did not properly remember all details about DR studies.

Risk

Most interviewed parents based their decision on study participation on the perceived risk of the DR study instead of on the prospect of direct benefit. However, parents frequently perceived risk differently than how it was categorized by the ERC.

How risk associated with specific study procedures was perceived also differed among parents: procedures that some parents considered minimal risk were considered risky by others. However, parents agreed that if there was risk associated with a specific study, parental consent is required.

Parents reported various factors that contributed to perceived risk. In general, parents considered their infants' participation in research often as riskier than participating in research themselves. Furthermore, some 
parents perceived a new study riskier than a study that was already running for several months. Also, anticipated regret added perceived risk, meaning that when regret could not be eliminated or reduced during the decisionmaking process, participating in research was perceived riskier. Having an "escape" for the study intervention, e.g., the possibility of unblinding the RFM if needed, reduced perceived risk.

\section{Discussion}

Obtaining ethically valid proxy consent for participation in DR studies from parents facing an imminent premature birth can be challenging. Despite being in an emotional and stressful situation, most interviewed parents in our study considered being approached for DR studies antenatally as a positive experience. According to parents, this was mostly due to appropriate timing and communication, compassion, and investigators not being obtrusive. Parents generally based decisions about study participation on perceived risk. Parents differed widely in how risks of specific study interventions were perceived, but agreed on the fact that consent is needed for DR studies that involve risk. There was no consensus on deferred consent for DR studies running at our NICU; however, most interviewed parents considered deferred consent appropriate for observational studies. Furthermore, when parents recalled information provided to them during the consent process, parental misunderstanding of various aspects of DR studies was frequently identified.

It has been advocated to give parents a voice in the discussion about consent for neonatal trials [13-15]. Earlier, McCarthy et al. [16] reported parental perspectives on consent for hypothetical neonatal trials. This study surveyed 600 parents and showed that parents feel they should play a central role in research involving their infants and that the acceptability of different consent approaches differed. Furthermore, Sloss et al. [17] reported parental perspectives on deferred consent. This study showed that the majority of 100 interviewed parents considered a deferred consent approach appropriate. With our study, we add to their findings by providing meaning and in-depth understanding of parental perspectives. Although this was a qualitative study and experiences of interviewed parents thus cannot be generalized, parents highlighted some important themes that may help to improve the validity of consent for DR studies.

For decades, the validity of consent for neonatal research has been questioned due to the fact that parents are

Consent for Delivery Room Studies: What

Can Be Learned from Parents approached in a particularly difficult time with often a tight timescale for decision-making [18]. Obtaining valid consent for DR studies may even be more complicated due to the medical condition of the mother. In our experience, investigators can therefore be reluctant to approach parents for DR studies. However, most interviewed parents considered approaching parents for DR studies antenatally appropriate, but reported that timing, communication, compassion, and not being obtrusive are keys for a positive experience. Addressing these factors when training research personnel may improve the validity of antenatal consent.

Approaching parents in an appropriate moment may furthermore improve the validity of consent, as parents may be more emotionally competent for decision-making about their infant's research participation. Parents in our study were approached several hours up to several days before delivery, and all considered this timing appropriate. Collaborating with the neonatal and obstetric staff helped our investigators to find the right moment to approach parents for study participation. However, more research is needed to define the most appropriate timing. Also the degree of investigators' compassion may influence the validity of parental consent, as compassion supports a better exchange of information, improves a trustful relationship, and may reduce emotional distress of parents [19]. Emphasizing the importance of compassion when training investigators in obtaining consent from parents may therefore help to improve the validity of parental consent. Furthermore, in our study, parents reported to appreciate how information on research was communicated; however, when interviewing parents, it was also noticed that elements of research were frequently forgotten, misunderstood, or misperceived. Parental difficulties to understand, comprehend, and recall elements of research were reported extensively [20]. Continued efforts should therefore be on improving information provision about DR studies. This may include more public education about research methodology, including concepts of randomization. However, misunderstanding may be a result of many different and possibly interacting sources, including inappropriate comprehension, as well as the usage of inappropriate vocabulary [21]. Therefore, training research personnel in appropriate communication is important. By making sure that language used is understood and by re-wording information according to parental needs, consent can be more valid. Another important topic for training is how to communicate that declining study participation is appropriate. In our study, parents reported not to feel pressurized in study partici- 
pation, but these feelings were reported by Richards et al. [22]. By avoiding obtrusiveness of investigators, consent can become more voluntarily, thus more valid.

Although parents considered being approached for consent for DR studies valuable, alternative approaches to consent were also suggested. Some parents stated it might be valuable to be informed on DR studies earlier in pregnancy, for instance, at a midwife's appointment. Parents described a process similar to the "blanket prenatal opt-out consent" as described by Janvier et al. [13]. This approach implies that parents are approached by a midwife/obstetrician for a blanket opt-out consent for DR studies that went through rigorous scientific and ethics review. Like neonatal care providers that were interviewed in our earlier study [11], interviewed parents suggested the prenatal opt-out as a possible addition to improve the deferred consent approach, but doing so would not absolve investigators of informing parents of eventual study participation of their infant, nor of obtaining consent for the usage of already obtained data. In contrast, various parents reported that consent could fully be waived in the case of observational DR studies. Interestingly, according to prevailing legislation [23], observational studies do not qualify for the usage of deferred consent, as observational studies lack the prospect of direct benefit. Although parental perceptions provide insight into possible improvements of the deferred consent approach, it must be acknowledged that most interviewed parents were not actually approached for deferred consent. More insight into perceptions of parents that were approached for deferred consent is therefore needed.

Zupancic et al. [24] reported that in making consent decisions on behalf of their infant, parents are influenced by risk and benefit assessments, attitudes toward research, and the integrity of the consent process. These factors were all reported in our study, with perceived risks as the most important factor. Interestingly, risks were perceived differently by parents and the ERC, as well as among parents. Providing more insight into how parents perceive risks of study interventions may help to understand why parents accept or decline study participation. Considerations about perceived risk, for instance, gained through community consultation, may inform risk classifications for DR studies, which may be used by investigators, providers, and ERCs, for instance, when deciding whether it is appropriate to use a deferred consent approach. Further research on perceived risks of study interventions of DR studies is therefore required.

Our findings should be seen in light of limitations. In our study, only 25 parents were interviewed using conve- nience sampling. Included parents may therefore not be representative to all parents. For example, all parents consented to at least 1 DR study, and only 1 bereaved parental dyad was included. As our study is an explorative qualitative study, we did not aim to generalize parental perspectives, but these limitations should be acknowledged. However, we argue that our study allowed us to determine areas to improve the validity of consent for DR studies. It furthermore revealed that obtained consent did not always meet all requirements for an ethically valid consent, yet parents did consider the consent procedure valid and valuable. Parents expressed the need for some sense of control of what happens to their infant especially in the case of interventions that they perceive as risky. These insights into parental perspectives may help to improve consent procedures for DR studies. However, more research is needed to further study parental perspectives, including more quantitative studies and studies focused on parents with different backgrounds.

\section{Conclusion}

Despite being in an emotional and stressful situation, parents in our study generally considered being approached for DR studies as a positive experience. According to interviewed parents, this was mostly due to appropriate timing and communication, compassion, and investigators not being obtrusive, factors that can all be addressed in training of research personnel. Consensus on perceived risk associated with study interventions and the appropriateness of deferred consent for DR studies running at our NICU was lacking. Interviewed parents expressed the need for consent for DR studies that they perceive as risky. Insight into parental perspectives may help to establish consent procedures that parents, research personnel, providers, and ethicists consider both valid and valuable.

\section{Acknowledgments}

We would like to thank all parents participating in this study.

\section{Statement of Ethics}

This study was reviewed by the Ethics Review Board of the Leiden University Medical Center. In concordance with laws and guidelines, a statement of no objection against execution of the study was issued (P16.316). Verbal consent was obtained. 


\section{Conflict of Interest Statement}

The authors have no conflicts of interest to declare.

\section{Funding Sources}

Arjan te Pas is a recipient of an NWO Innovational Research Incentives Scheme (VIDI 91716428).

\section{Author Contributions}

Maria den Boer: conceptualization, methodology, formal analysis, investigation, and writing - original draft; Mirjam Houtlosser: methodology, validation, formal analysis, and writing - original draft; Ruben Witlox: investigation and writing - review and editing; Henriëtte van Zanten: investigation and writing - review and editing; Martine de Vries: conceptualization, methodology, and writing - review and editing; Arjan te Pas: conceptualization, resources, writing - review and editing, and supervision. All authors approved the final manuscript as submitted and agree to be accountable for all aspects of the work.

\section{References}

1 World Medical Association. Declaration of Helsinki: ethical principles for medical research involving human subjects; 2013.

2 International Conference on Harmonization. Guideline for good clinical practice (CPMP/ ICH/135/95); 2006.

3 Beauchamp TL, Childress JF. Principles of biomedical ethics. USA: Oxford University Press; 2001.

4 Foglia EE, Owen LS, Kirpalani H. Delivery room research: when does poor quality evidence become an ethical issue? Pediatrics. 2015;135(5):e1368.

5 Woolfall K, Young B, Frith L, Appleton R, Iyer A, Messahel S, et al. Doing challenging research studies in a patient-centred way: a qualitative study to inform a randomised controlled trial in the paediatric emergency care setting. BMJ open. 2014;4(5):e005045.

6 Jansen-van der Weide MC, Caldwell PH, Young B, de Vries MC, Willems DL, Van't Hoff W, et al. Clinical trial decisions in difficult circumstances: parental consent under time pressure. Pediatrics. 2015;136(4):e98392.

7 Mason S. Obtaining informed consent for neonatal randomised controlled trials: an "elaborate ritual"? Arch Dis Child Fetal Neonatal Ed. 1997;76(3):F143-5.

8 Rich W, Finer NN, Gantz MG, Newman NS, Hensman AM, Hale EC, et al. Enrollment of extremely low birth weight infants in a clinical research study may not be representative. Pediatrics. 2012;129(3):480-4.

9 den Boer MC, Houtlosser M, Foglia EE, Tan RNGB, Engberts DP, te Pas AB. Benefits of recording and reviewing neonatal resuscitation: the providers' perspective. Arch Dis Child Fetal Neonatal Ed. 2018;104:F528-34.
10 den Boer MC, Houtlosser M, van Zanten HA, Foglia EE, Engberts DP, Te Pas AB. Ethical dilemmas of recording and reviewing neonatal resuscitation. Arch Dis Child Fetal Neonatal Ed. 2018 May;103(3):F280-4.

11 den Boer MC, Houtlosser M, Foglia EE, Lopriore E, de Vries MC, Engberts DP, et al. Deferred consent for delivery room studies: the providers' perspective. Fetal Neonatal. 2020;105(3):310-5.

12 den Boer MC, Houtlosser M, Witlox RSGM, van der Stap R, de Vries MC, Lopriore E, et al. Reviewing recordings of neonatal resuscitation with parents. Arch Dis Child Fetal Neonatal Ed. 2021.

13 Janvier A, Farlow B. The ethics of neonatal research: an ethicist's and a parents' perspective. Semin Fetal Neonatal Med. 2015;20(6): 436-41.

14 Janvier A, Bourque CJ, Dahan S, Robson K, Barrington KJ. Integrating parents in neonatal and pediatric research. Neonatology. 2019; 115(4):283-91.

15 den Boer MC, Houtlosser M, Foglia EE, Davis PG, van Kaam AH, Kamlin COF, et al. Deferred consent for the enrolment of neonates in delivery room studies: strengthening the approach. Arch Dis Child Fetal Neonatal Ed. 2019.

16 McCarthy KN, Ryan NC, O’Shea DT, Doran K, Greene R, Livingstone V, et al. Parental opinion of consent in neonatal research. Archives of disease in childhood fetal and neonatal edition. Fetal Neonatal. 2019 Jul;104(4): F409-14.
17 Sloss S, Dawson JA, McGrory L, Rafferty AR, Davis PG, Owen LS. Observational study of parental opinion of deferred consent for neonatal research; 2020.

18 Ballard HO, Shook LA, Desai NS, Anand KJ. Neonatal research and the validity of informed consent obtained in the perinatal period. J Perinatol. 2004;24(7):409-15.

19 Quaschning K, Körner M, Wirtz M. Analyzing the effects of shared decision-making, empathy and team interaction on patient satisfaction and treatment acceptance in medical rehabilitation using a structural equation modeling approach. Patient Educ Couns. 2013;91(2):167-75

20 Dawson A, Spencer SA. Informing children and parents about research. Arch Dis Child. 2005;90(3):233-5.

21 Ali M. Communication skills 2: overcoming barriers to effective communication. Nursing Times. 2017;114(1):40-2.

22 Richards J, Rankin J, Juszczak E, Dorling J, McGuire W, Embleton ND. Parental experiences of being approached to join multiple neonatal clinical trials: qualitative study PARENT. Arch Dis Child Fetal Neonatal Ed. 2020.

23 European Parliament, Council of the European Union. Regulation (EU) No 536/2014 of the European Parliament and of the Council of 16 April 2014 on clinical trials on medicinal products for human use, and repealing Directive 2001/20/EC. Off J Eur Un. 2014;L158:176.

24 Zupancic JA, Gillie P, Streiner DL, Watts JL, Schmidt B. Determinants of parental authorization for involvement of newborn infants in clinical trials. Pediatrics. 1997;99(1):e6. 